\title{
Potential immune escape mechanisms underlying the distinct clinical outcome of immune checkpoint blockades in small cell lung cancer
}

\author{
Yaru Tian ${ }^{1}$, Xiaoyang Zhai ${ }^{2,3}$, Anqin $\mathrm{Han}^{3}$, Hui Zhu ${ }^{3,1^{*}}$ and Jinming $\mathrm{Yu}^{3,1^{*}}$
}

\begin{abstract}
Small cell lung cancer (SCLC) is one of the deadliest cancer types in the world. Despite the high response rate to frontline platinum-containing doublets, relapse is inevitable for the majority of patients and the prognosis is poor. Topotecan, which has limited efficacy, has remained the standard second-line therapy for approximately three decades. Although SCLC has a high mutation burden, the clinical efficacy of immune checkpoint blockades (ICBs) in SCLC is far less pronounced than that in non-small cell lung cancer (NSCLC). Only atezolizumab in combination with chemotherapy improved overall survival over chemotherapy alone in the phase III CheckMate 133 trial and has recently received FDA approval as first-line therapy. Most studies concerning ICBS in SCLC are limited to early-phase studies and found that ICBs were not superior to traditional chemotherapy. Why is there such a large difference between SCLC and NSCLC? In this review, comparative analyses of previous studies indicate that SCLC is even more immunodeficient than NSCLC and the potential immune escape mechanisms in SCLC may involve the low expression of PD-L1 and the downregulation of major histocompability complex (MHC) molecules and regulatory chemokines. In consideration of these immune dysfunctions, we speculate that chemotherapy and radiotherapy prior to immunotherapy, the combination of ICBs with antiangiogenic treatment, and selecting tumor mutation burden in combination with PD-L1 expression as biomarkers could be promising strategies to improve the clinical efficacy of immunotherapy for SCLC.
\end{abstract}

Keywords: SCLC, Immune checkpoint blockades, Immune escape mechanisms

\section{Background}

Worldwide, lung cancer is the leading cause of cancer incidence and mortality, with 2.1 million new cases and 1.8 million deaths estimated in 2018, representing $18.4 \%$ of the total cancer-related deaths [1]. Small cell lung cancer (SCLC) accounts for approximately 14\% of all lung cancers $[2,3]$ and is highly aggressive and lethal, characterized by a rapid growth rate and early development of widespread metastasis. Most patients with SCLC have a history of cigarette smoking [4-6] and harbor a high prevalence of somatic mutations [7-10].

\footnotetext{
*Correspondence: drzhuh@126.com; sdyujinming@163.com

${ }^{3}$ Department of Radiation Oncology, Shandong Cancer Hospital and Institute, Shandong First Medical University and Shandong Academy of Medical Sciences, 440 Jiyan Road, Jinan 250117, China

Full list of author information is available at the end of the article
}

SCLC is classified as limited-stage SCLC (LS-SCLC) and extensive-stage SCLC (ES-SCLC). ES-SCLC, generally with distant metastasis at the time of diagnosis, accounts for approximately two thirds of all SCLC. Platinum-based chemotherapy remains the first-line standard of care for SCLC and the response rate could reach more than $70 \%$. Although SCLC is highly responsive to initial therapy, most patients with SCLC will inevitably relapse. Consequently, the prognosis of SCLC is rather poor, with an estimated 2-year overall survival (OS) of less than $5 \%$ [11-15]. Currently, topotecan is the only drug approved by the US Food and Drug Administration (FDA) as a second-line therapy. However, the efficacy of topotecan is limited, with a response rate of approximately $25 \%$ for platinum-sensitive patients and less than $10 \%$ for platinum-resistant or refractory patients [16-18].

(c) The Author(s). 2019 Open Access This article is distributed under the terms of the Creative Commons Attribution 4.0 International License (http://creativecommons.org/licenses/by/4.0/), which permits unrestricted use, distribution, and 
Other recommended second-line options included irinotecan, vinorelbine, gemcitabine, and temozolomide [11]. Clinical benefit is even dismal for treatment beyond the second line. Contrasting sharply with non-small cell lung cancer (NSCLC), SCLC is a hard-to-treat cancer with lack of progress for nearly 30 years.

Immunotherapy has revolutionized the standard of care across multiple cancer types. As tumors develop, they can escape immune surveillance by various mechanisms in steps in the cancer-immunity cycle $[19,20]$. Immune checkpoints, limiting antitumor responses and contributing to immune escape, have been readily confirmed as negative regulators in recent years. Two such checkpoints, cytotoxic T-lymphocyte protein 4 (CTLA4) and programmed cell death protein-1 (PD-1), are the best [21]. The co-inhibitory receptor CTLA-4, which is expressed on T cells, can outcompete CD28 to bind to CD80 and CD86 on APCs preventing both the activation and proliferation of $\mathrm{T}$ cells in the lymphnodes [22, 23]. Moreover, the highly constitutively expressed CTLA-4 on Treg cells also mediates the suppressive role of Treg cells. Anti-CTLA4 blockades significantly improve the overall survival of patients with advanced melanoma [24, 25]. Differing from CTLA4 in its mechanism of action, the PD-1/PD-L1 pathway mainly mediates $T$ cell dysfunction in the tumor microenvironment (TME). PD-1 is induced on activated $\mathrm{T}$ cells through TCR and co-stimulatory signals. These effector $\mathrm{T}$ cells are capable of recognizing tumor neoantigens and then producing interferon$\gamma$ (INF- $\gamma$ ), thereby inducing the expression of PD-L1 on tumor cells and stromal cells. Ultimately, the interaction between PD-L1 and PD-1 disrupts the antitumor activity of effector T cells. This local immune escape mechanism has been termed "adaptive immune resistance" [26-29]. Sanmamed and Chen [30] have confirmed anti-PD/PD-L1 therapy as a clearest approach to the normalization of cancer immunotherapy; it can selectively repair the tumor-induced immune defect and restore immune activity in the TME without general immune activation [30]. Patients with a variety of cancer types have exhibited promising clinical benefit/risk ratio from anti-PD-1/PDL1 therapies, resulting in FDA approval for the corresponding indications $[21,26,30,31]$.

The progress made in uncovering the biology of SCLC and its microenvironment has offered new therapeutic strategies for SCLC [32]. The high mutation burden, which produces a large number of potential tumorspecific antigens, raised hope regarding immunotherapy in SCLC [33]. In this review, we mainly illustrate the rationale behind immunotherapy for SCLC, the differences in clinical outcomes of ICBs and the underlying mechanisms in NSCLC and SCLC. Finally, we propose some strategies to improve the clinical outcome of immunotherapy for SCLC.

\section{Rationale of immunotherapy therapy for SCLC}

Nearly $98 \%$ of SCLC patients have a smoking history [4-6]. Tobacco can exert carcinogenic effects through more than 60 chemicals that are capable of binding and mutating DNA [10]. Consequently, SCLC has a high tumor mutation burden (TMB) of 8.62 nonsynonymous or missense mutations per million base pairs among various solid tumors [7-10]. These mutations can therefore generate neoantigens (new protein or peptide sequences) that will be presented by MHC molecules and recognized by $\mathrm{T}$ cells $[20,34,35]$. Immunogenic neoantigens that elicit tumorspecific $\mathrm{CD} 8+\mathrm{T}$ cell activities can thereby enhance the clinical benefits of immune checkpoint blockades (ICBs) $[36,37]$. The TMB has been confirmed as an effective biomarker to predict the clinical outcome of ICBs in many tumors [35, 38]. Moreover, there is a similar level and distribution of TMBs between NSCLC and SCLC, and similar to in NSCLC, the TMB has been associated with the clinical outcome of ICBs in SCLC [33, 39].

Moreover, retrospective findings indicated that host immune status was strongly associated with the prognosis of SCLC. The inflammatory status of the host immune system was suggestive of a beneficial clinical outcome. Immunologic indicators associated with better prognosis included tumor-infiltrating macrophages (TIMs) and lymphocytes (TILs) [40-42], effective CD4+ $\mathrm{T}$ cells including Th17 cells [43], a high effector $\mathrm{T}$ cell (Teff) to regulatory $\mathrm{T}$ cell (Treg) ratio [43], a high neutrophil to lymphocyte ratio (NLR) and a high platelet to lymphocyte ratio (PLR) [44]. In addition, suppressive immune features indicating a poor prognosis of SCLC include the frequency of CD14+HLA-DR-/low myeloidderived suppressor cells (MDSCs) [45], the C-reactive protein/albumin (CRP/Alb) ratio[46] and a higher Treg cell ratio in tumor infiltrates [47]. These clinical evidence further illustrated the significance of the immune response and the possibility of immunotherapy for SCLC.

In conclusion, the high TMB and the status of the host immune system may hold new promise for immunotherapy for SCLC. Among multiple immunotherapy strategies, ICBs have achieved great success in treating SCLC.

\section{Clinical outcomes of ICBs in NSCLC and SCLC}

Immune checkpoint blockades, including anti-CTLA4 antibody (ipilimumab), anti-PD-1 antibodies (pembrolizumab and nivolumab), and anti-PD-L1 antibodies (atezolizumab and durvalumab), have showed remarkable and durable responses across multiple cancer types and received FDA approval $[21,26,30,31]$. Table 1 summarizes the major clinical trials involving ICBs in NSCLC and SCLC.

\section{First-line}

First, Reck et al. conducted a randomized phase II trial to investigate ipilimumab in combination with chemotherapy 


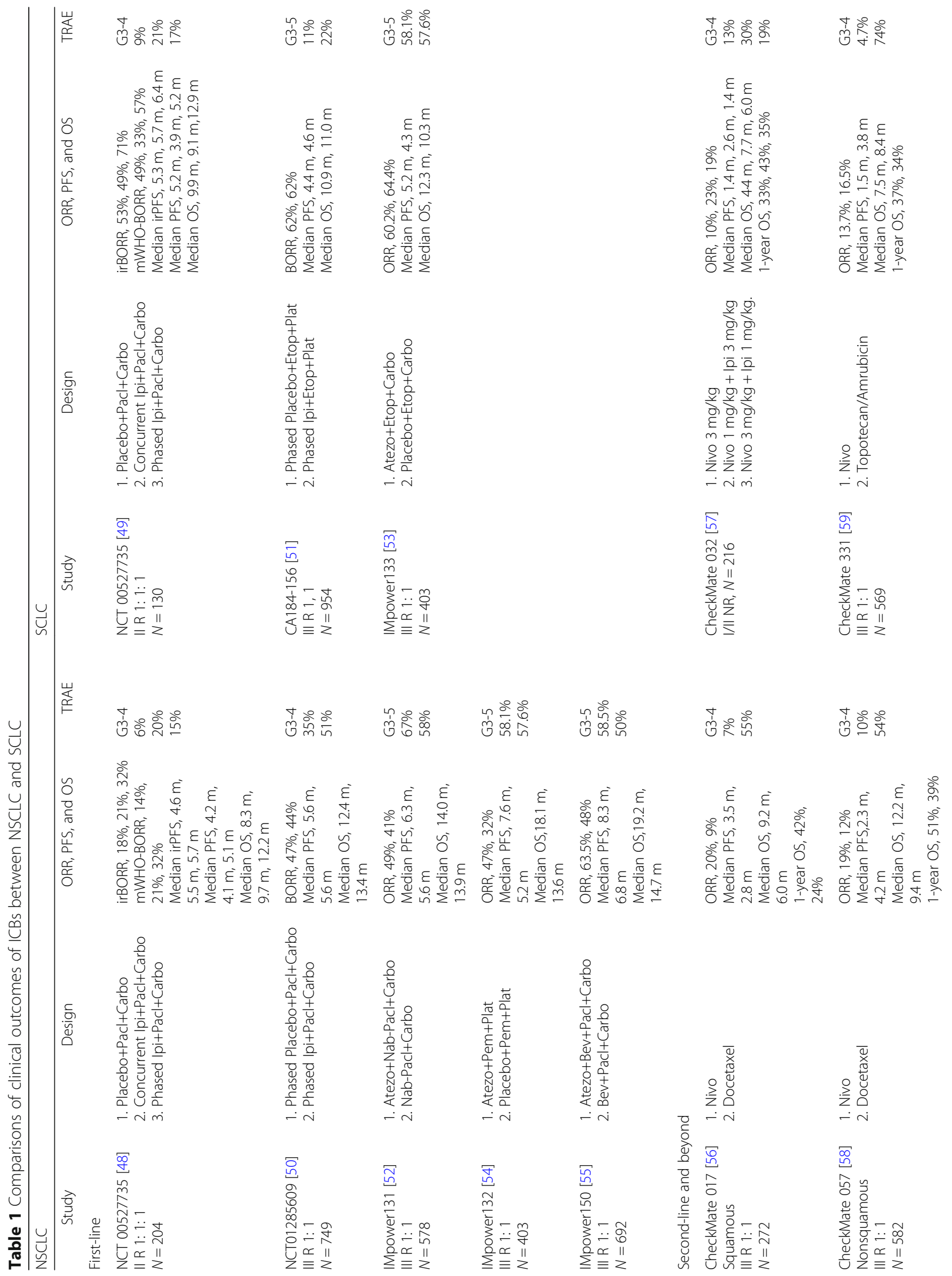


Than et al. Journal of Hematology \& Oncology $\quad$ (2019) 12:67

Page 4 of 12

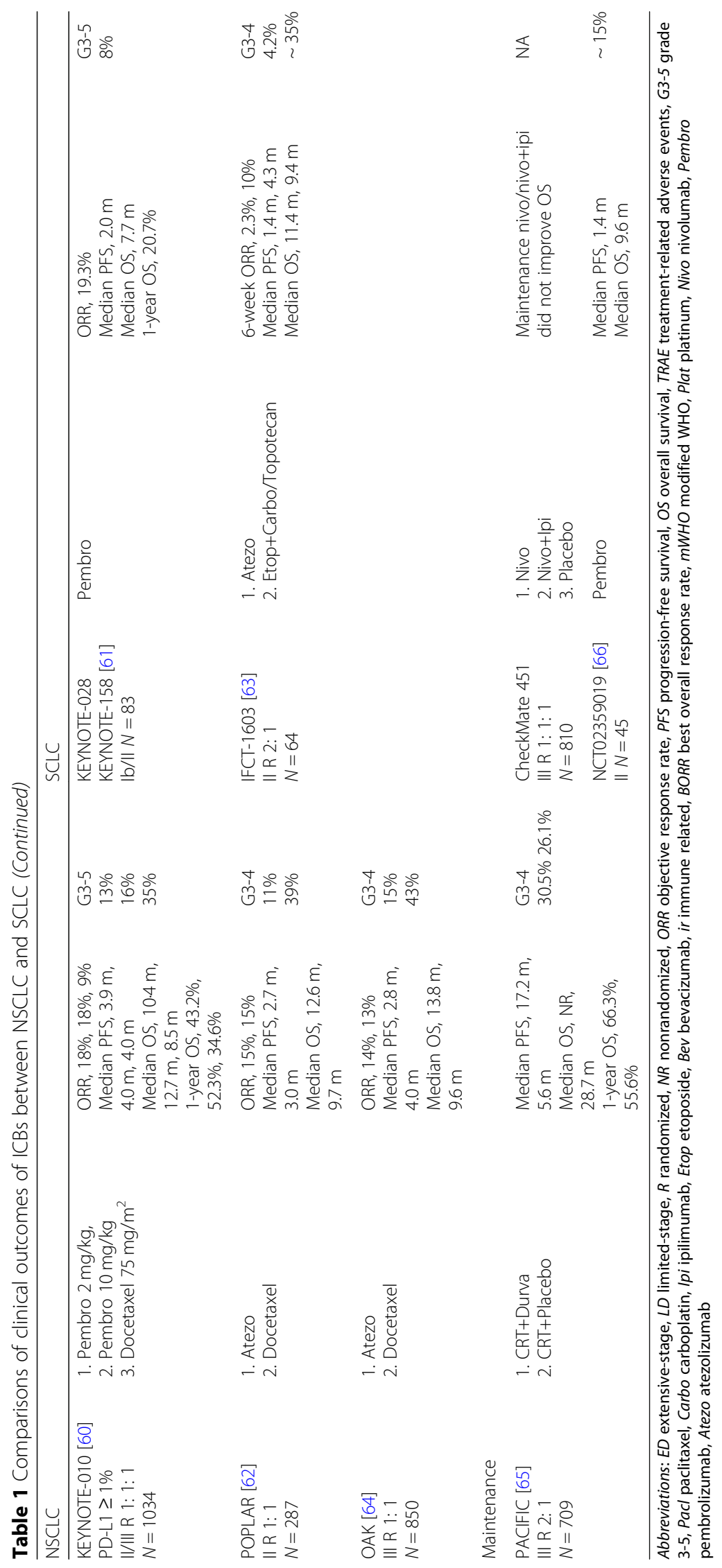


in previously untreated patients with lung cancer (ED-SCLC, $n=130$; NSCLC, $n=204$ ) [48, 49]. For both the NSCLC and SCLC cohorts, phased ipilimumab with carboplatin and paclitaxel but not a concurrent regimen showed improved immune-related (ir) progression-free survival (PFS) and a numeric, though not statistically significant, increase in median overall survival (OS) over chemotherapy alone. Overall grade 3-4 (G3-4) immune-related adverse events (irAEs) were more common for phased ipilimumab. Later, the phase III CA184-156 study further evaluated the efficacy of phased ipilimumab with etoposide and platinum as the first-line regimen for ED-SCLC [51]. Disappointingly, phased ipilimumab did not significantly prolong PFS and OS over the placebo and produced more irAEs. The rate of treatment-related discontinuation was even higher $(18 \%$ v $2 \%)$. Similar results were observed in patients with advanced squamous NSCLC [50]. Another phase II trial involving phased ipilimumab to first-line chemotherapy even reported a G3-5 irAE rate as high as $69.2 \%$ with 5 of 42 patients dying [67]. The unfavorable benefit to risk profile limits the first-line application of ipilimamab in lung cancer.

The addition of atezolizumab to chemotherapy as the first-line treatment significantly improved the ORR and PFS among patients with metastatic nonsquamous and squamous NSCLC in IMpower 131 and IMpower 132 $[52,54]$. Among patients with metastatic nonsquamous NSCLC, atezolizumab plus bevacizumab and chemotherapy significantly improved PFS and OS in IMpower 150 , which led to FDA approval of the combined regimen for the first-line treatment of nonsquamous NSCLC [55]. For ED-SCLC, the clinical efficacy and safety of atezolizumab plus chemotherapy was evaluated in IMpower133 [53]. The interim analysis of the intention-to-treat population in this phase III trial showed that the addition of atezolizumab to standard chemotherapy significantly prolonged OS and PFS over the placebo (median OS, $12.3 \mathrm{v} 10.3$ months and median PFS, $5.2 \mathrm{v}$ 4.3 months) and increased the 1-year OS by $13.5 \%$ (51.7\% v 38.2\%). The safety profile was consistent with previous observations. In March 2019, this combined regimen was approved as a first-line therapy for ED-SCLC. Overall, atezolizumab in combination with chemotherapy as a first-line treatment could be a novel option for people with advanced NSCLC and ED-SCLC.

Pembrolizumab in combination with standard chemotherapy resulted in significantly prolonged OS and PFS compared with chemotherapy alone among patients with squamous and nonsquamous NSCLC in KEYNOTE-407 and KEYNOTE-189 [68, 69]. However, the attempt to use pembrolizumab in the first-line setting for SCLC is limited. Only the phase Ib PembroPlus trial indicated that the standard dose pembrolizumab could be safely combined with many chemotherapy regimens across 6 advanced solid tumors, including relapsed SCLC [70]. A randomized phase III trial, KEYNOTE-604, is underway to assess the clinical benefit of pembrolizumab in combination with etoposide and platinum as a first-line treatment for ES-SCLC (Table 2).

\section{Second-line}

As shown in Table 1, the second-line nivolumab monotherapy significantly improved ORR, PFS, and OS compared with docetaxel among patients with advanced squamous and nonsquamous NSCLC in CheckMate 017 and CheckMate $057[56,58]$. The response rate to nivolumab monotherapy was approximately twice that of docetaxel $(20 \% \mathrm{v} 10 \%)$, and nivolumab extended OS by approximately 3 months over chemotherapy. For SCLC, in the nonrandomized cohort in CheckMate 032 [57], the ORR was $10 \%$ (10 of 98 ) and $23 \%$ (14 of 61), and the median OS was 4.4 and 7.7 months for patients receiving nivolumab $3 \mathrm{mg} / \mathrm{kg}$ and nivolumab $1 \mathrm{mg} / \mathrm{kg}$ plus ipilimumab $3 \mathrm{mg} / \mathrm{kg}$, respectively. One-year OS was 33\% and $43 \%$ for the two groups, respectively. Based on this trial, nivolumab and nivolumab plus ipilimumab were added as category $2 \mathrm{~A}$ recommendations to the NCCN guidelines [11]. In August 2018, under accelerated approval, FDA approved nivolumab for treating patients with relapsed SCLC after the failure of platinum-based chemotherapy and one or more other lines of treatment. Unfortunately, CheckMate 331, a randomized phase III trial, demonstrated that nivolumab was inferior to topotecan or amrubicin in improving ORR, PFS, and OS among patients with relapsed SCLC [59].

Based on KEYNOTE-010, pembrolizumab was approved as a second-line treatment for advanced NSCLC patients with PD-L1 expression on $\geq 1 \%$ of tumor cells [60]. The phase Ib KEYNOTE-028 trial showed favorable efficacy and tolerable safety of pembrolizumab in treating patients with relapsed ED-SCLC and PD-L1 expression on $\geq 1 \%$ of tumor and stromal cells [71]. Further, the phase II KEYNOTE-158 trial confirmed the beneficial role of pembrolizumab in treating SCLC [72]. The latest results of KEYNOTE-028 and KEYNOTE-158 from 2019 from the American Association for Cancer Research (AACR) showed that pembrolizumab produced a durable response with tolerable toxicity for advanced SCLC patients after $\geq$ 2 lines of prior therapy. The ORR was $19.6 \%$ (16 of 83 ), with 2 patients having a complete response $(\mathrm{CR})$ and 14 having a partial response (PR). More than half (9 of 16) had a response duration of $\geq 18$ months. The median PFS was 2.0 months, and the median OS was 7.7 months, with a 1-year OS rate of $20.7 \%$. The toxicity was manageable, with a G3-5 AE incidence of 9\% [61]. Despite the encouraging results of single-arm studies, large randomized controlled studies are needed. 
Table 2 Ongoing clinical trials of immune checkpoint blockades in SCLC

\begin{tabular}{|c|c|c|c|c|c|c|c|}
\hline Study & Stage & $\mathrm{Ph}$ & Pts. & Design & Primary end point & NCT number & Primary completion \\
\hline \multicolumn{8}{|l|}{ First-line } \\
\hline KEYNOTE-604 & ED & III & 453 & Pembro+EP $\vee$ Placebo+EP & PFS, OS & NCT03066778 & December 2019 \\
\hline REACTION & ED & $\|$ & 118 & Pembro+EP $\vee$ EP & 6-month PFS & NCT02580994 & August 2020 \\
\hline NA & LD/ED & I & 80 & Pembro+EP \pm RT & MTD & NCT02402920 & July 31, 2023 \\
\hline NA & LD & II / III & 506 & Atezo+EP+RT vEP+RT & PFS, OS & NCT03811002 & May 2024 \\
\hline CASPIAN & ED & III & 988 & Durva \pm Treme+EP $\vee$ EP & PFS, OS & NCT030s43872 & September 2019 \\
\hline CLOVER & $L D^{b}$ & I & 300 & Durva \pm Treme+EP & $\mathrm{DLT} ; \mathrm{AE}$ & NCT03509012 & April 2022 \\
\hline PAVE & ED & ॥ & 55 & Avelumab+EP & 1- year PFS & NCT03568097 & November 2020 \\
\hline \multicolumn{8}{|c|}{ Second-line and beyond } \\
\hline BLOLUMA & LD/ED ${ }^{a}$ & $\|$ & 106 & Nivo+lpi & ORR & NCT03083691 & March 2019 \\
\hline MCC-19163 & LD/ED & $\|$ & 41 & Nivo+Ipi+Ad.p53-DC vaccine & DCR & NCT03406715 & April 2020 \\
\hline CA209-9YT & ED & $\|$ & 40 & Nivo+Ipi & Teff/Treg & NCT03670056 & September 2020 \\
\hline NA & ED & $\|$ & 29 & Nivo+Gemcitabine & ORR & NCT03662074 & September 2020 \\
\hline AFT-17 & LD/ED & $\|$ & 98 & Pembro v Topotecan & PFS & NCT02963090 & May 2019 \\
\hline KEYNOTE PN758 & ED & |/ $\|$ & 84 & Pembro+Pegzilarginase & $\mathrm{AE}, \mathrm{ORR}$ & NCT03371979 & December 2020 \\
\hline NA & LD/ED & $\|$ & 80 & Pembro+EP $\pm R T$ & PD-L1 & NCT02934503 & October 2019 \\
\hline ML39728 & LD/ED & $\|$ & 35 & Atezo & ORR & NCT03262454 & December 2019 \\
\hline Winship3112-15 & LD/ED & ॥ & 28 & Durva \pm Treme+RT v RT & PFS, ORR & NCT02701400 & January 2020 \\
\hline PASSION & ED & $\|$ & 135 & SHR-1210+Apatinib & $\mathrm{AE}, \mathrm{ORR}$ & NCT03417895 & March 2019 \\
\hline \multicolumn{8}{|l|}{ Maintenance } \\
\hline STIMULI & LD & $\|$ & 260 & Nivo+Ipi v Obsesrvation & PFS, OS & NCT02046733 & October 2019 \\
\hline CA209-840 & ED & |/ II & 21 & Nivo+lpi+RT & PFS & NCT03043599 & April 2021 \\
\hline ACHILES & LD & $\|$ & 212 & Atezo v Observation & 2-year OS & NCT03540420 & December 2023 \\
\hline NA & LD & $\|$ & 51 & Durva+EP+RT & PFS & NCT03585998 & June 2021 \\
\hline ADRIATIC & LD & III & 600 & Durva \pm Treme v Placebo & PFS, OS & NCT03703297 & June 2021 \\
\hline
\end{tabular}

Abbreviations: $E D$ extensive-stage, $L D$ limited-stage, $a$ including NSCLC, $b$ including 3 other solid tumors, ORR objective response rate, $P F S$ progression-free survival, OS overall survival, $A E$ adverse event, Nivo nivolumab, Ipi ipilimumab, RT radiotherapy, Pem pembrolizumab, EP etoposide plus platinum, MTD maximum tolerated dose, Atezo atezolizumab, Durva durvalumab, Treme tremelimumab, DLT dose-limiting toxicities, NA not available

Atezolizumab also significantly improved OS by 3 to 4 months over docetaxel in patients with previously treated NSCLC in POPLAR and OAK [62, 64]. Unfortunately, the randomized phase II IFCT-1603 trial indicated that atezolizumab was not superior to chemotherapy as a secondline treatment in SCLC [63].

\section{Maintenance}

Durvalumab, an anti-PD-L1 antibody, has significantly prolonged PFS by more than twofold compared with the placebo (17.2 v 5.6 months, HR=0.51) among patients with unresectable stage III NSCLC without disease progression after concurrent chemoradiotherapy in PACIFIC [65]. For patients with SCLC, the clinical outcome of ICBs as a maintenance regimen is quite dissatisfactory. A single-group, phase II study showed that maintenance pembrolizumab did not prolong OS compared with historical chemotherapy after first-line chemotherapy in patients with ED-SCLC [66]. In November 2018,
Bristol-Myers Squibb announced that the phase III CheckMate 451 trial did not meet the primary endpoint of OS with nivolumab and nivolumab plus ipilimumab versus the placebo as maintenance therapy in patients with ED-SCLC.

In conclusion, anti-PD-1/PD-L1 blockades have revolutionized the standard treatment for NSCLC across the first-line, second-line, and maintenance settings based on multiple large randomized controlled trials (Table 1). However, for SCLC, despite moderate benefit in phase I/II trials, ICBs did not outcompete traditional chemotherapy in large randomized trials. Only atezolizumab in combination with chemotherapy conferred a survival benefit in CheckMate 331. Table 2 lists the ongoing trials involving ICBs or combination regimens in SCLC.

\section{Potential mechanisms underlying discrepant outcomes of ICBs between SCLC and NSCLC}

SCLC, with a high TMB similar to that of NSCLC, is theoretically likely to respond to immunotherapy. 
However, the clinical outcome of ICBs in SCLC seems far less effective than in NSCLC. There is a need to define the underlying differences in immune regulation patterns between SCLC and NSCLC to further guide immunotherapy for SCLC.

\section{Low PD-L1 expression in SCLC}

PD-L1 expression reflects pre-existing antitumor immunity in the TME and has been associated with a clinical benefit from anti-PD-1/PD-L1 blockade across multiple cancer types, including NSCLC [73-75]. PD-L1 expression in NSCLC is approximately $50-70 \%$ according to previous reports $[55,56,58,62,64,68,69,76]$. In stark contrast to NSCLC, PD-L1 expression in SCLC has been reported to be relatively low in most studies, as listed in Table 3. Most studies showed less than 50\% PD-L1 expression in SCLC. The reason for the disparity in PD-L1 expression in these findings is not well understood and may be explained by the differences in staining antibodies, scoring algorithms, tissue biopsy types (archival or fresh), and detection platforms (Table 3). Notably, substantial PD-L1 expression occurs on stroma cells, including tumor-infiltrating immune cells (TILs and TIMs), in SCLC and less in tumor cells. Some of these studies demonstrated a positive correlation between PD-L1 expression on TICs and a favorable clinical outcome for SCLC patients [41, 66, 77, 80, 81]. Emerging data indicated that infiltrating stroma cells, such as dendritic cells and macrophages, could have protumorigenic functions by shaping antitumor immunity and the response to immunotherapy [84-86]. Perhaps PD-L1 expression in immune cells may play a exceptional role in the pathophysiological process in SCLC. Overall, the relatively low PD-L1 expression may be at least one reason that the efficacy of ICBs in SCLC is not as good as that in NSCLC.

\section{Downregulation of MHC molecules in SCLC}

The downregulation of $\mathrm{MHC}$ molecules is an immune escape mechanism. In contrast to NSCLC cells, which readily express MHC class I molecules, most SCLC cell lines and tissues showed marked deficient expression of MHC class I molecules, thus directly preventing tumor cells from presenting neoantigens to $\mathrm{CD} 8+\mathrm{T}$ cells in the lymph nodes and inhibiting CTL recognition in the TME [87, 88]. Additionally, due to the lack of IFN- $\gamma$ inducible expression of class II transactivator (cIITA), MHC class II molecule expression is absent in SCLC cells and significantly lower in SCLC TILs compared with in NSCLC, resulting in reduced presentation of tumor neoantigens to CD4+ T cells. In addition, mainly induced by IFN- $\gamma$-producing $\mathrm{T}$ cells, MHC class II

Table 3 PD-L1 expression in SCLC

\begin{tabular}{|c|c|c|c|c|c|c|}
\hline Author & Patients $^{c}$ & IHC assay & Biopsy types & TC PD-L1 cutoff & Cell type & PD-L1 expression \\
\hline Antonia et al. [57] & 146 & 28-8/Dako & Archival or fresh & $\begin{array}{l}1 \% \\
5 \%\end{array}$ & Tumor cell & $\begin{array}{l}16.4 \% \\
4.8 \%\end{array}$ \\
\hline Ott et al. [71] & 145 & 22C3/Dako & Archival or fresh & $1 \%$ & $\begin{array}{l}\text { Tumor and } \\
\text { Stromal cell }\end{array}$ & $31.7 \%$ \\
\hline Hyun et al. [72] & 92 & 22C3/Dako & Archival or fresh & $1 \%$ & Tumor and stromal cell & $45.7 \%$ \\
\hline Inamura et al. [77] & 74 & E1 L3N/CST & Archival & $5 \%$ & Tumor cell & $18.9 \%$ \\
\hline Yasuda et al. [78] & 39 & 22C3/Dako & Archival & $1 \%$ & Tumor cell & $2.5 \%$ \\
\hline Pujol et al. [63] & 53 & SP142Nentana & Archival & $1 \%$ & Tumor and stromal cell & $2 \%$ \\
\hline Gadgeel et al. [66] & $\begin{array}{l}30 \\
20\end{array}$ & 22C3/Dako & Archival & $1 \%$ & $\begin{array}{l}\text { Tumor cell } \\
\text { Stromal cell }\end{array}$ & $\begin{array}{l}10 \% \\
40 \%\end{array}$ \\
\hline Schultheis et al. [79]. & $94^{d}$ & $\begin{array}{l}\text { 5H1/Chen's lab } \\
\text { E1 L3N/CST }\end{array}$ & Archival & $1 \%$ & $\begin{array}{l}\text { Tumor cell } \\
\text { Stromal cell }\end{array}$ & $\begin{array}{l}0.0 \% \\
\text { TIMs, } 18.5 \% \\
\text { TILs, } 48 \%\end{array}$ \\
\hline Berghoff et al. [41] & 32 & NA/Dako & Archival & $5 \%$ & $\begin{array}{l}\text { Tumor cell } \\
\text { Stromal cell }\end{array}$ & $\begin{array}{l}34.4 \% \\
\text { TIMs, } 28.1 \% \\
\text { TILs, } 25.0 \%\end{array}$ \\
\hline Yu et al. [80] & $194^{e}$ & $\begin{array}{l}\text { SP142Nentana } \\
\text { 28-8/Dako }\end{array}$ & Archival & $1 \%$ & $\begin{array}{l}\text { Tumor cell } \\
\text { Stromal cell }\end{array}$ & $\begin{array}{l}16.5 \% \\
\text { TILs, } \\
56.2 \%, 44.8 \%\end{array}$ \\
\hline Kim et al. [81] & 120 & $\begin{array}{l}\text { MAB1561/ } \\
\text { R\&D system }\end{array}$ & Archival & $1 \%$ & $\begin{array}{l}\text { Tumor cell } \\
\text { Stromal cell }\end{array}$ & $\begin{array}{l}14.2 \% \\
\text { TILs, } 23.3 \%\end{array}$ \\
\hline Komiya et al [82] & 99 & EPR1161/Abcam & Archival & $5 \%$ & Tumor cell & $82.8 \%$ \\
\hline Ishii et al. [83] & 102 & NA/Abcam & Archival & $5 \%$ & Tumor cell & $71.6 \%$ \\
\hline
\end{tabular}

Abbreviations: $c$ patients who are PD-L1 evaluable, $d$ small cell carcinomas 94 patients (SCLC 61; extrapulmonary 33), e total 194 patients, LD-SCLC 98,ED-SCLC 96, IHC immunohistochemistry, TC tumor cell, CST cell signaling technology, TIMs tumor infiltration macrophage, TILs tumor-infiltrating lymphocytes, NA not available* 
molecules also reflect a less immunogenic environment in SCLC compared with NSCLC [89-91].

\section{Immunosuppression induced by SCLC cells}

The interaction between SCLC cells and the immune system seems to be more complicated than previously thought. Tumors may destroy the host immune system in a variety of ways. The functions of the immune system, including lymphocyte reactivity to lectins and cytokines and the production of cytokines, were even more impaired in patients with SCLC compared with patients with NSCLC. The peripheral blood lymphocytes (PBLs) of SCLC patients showed significantly lower proliferative responses to phytohemagglutinin and human recombinant interleukin 2 (IL-2) than those of the NSCLC and noncancer groups. The ability of PBL to produce lymphokines (IL-2 and macrophage-activating factor) was evidently impaired in the SCLC group but not in the NSCLC group [92]. In contrast to NSCLC, the suppression of cytokine secretion in SCLC was dependent on tumor load and could be improved upon the reduction of the tumor load [93]. In addition, Wang et al. found that IL-15 secreted by SCLC cells could contribute to local and systemic immune escape and poor prognosis by inhibiting CD4+ $\mathrm{T}$ cell proliferation and supporting Treg cell induction [47]. Moreover, CD47 mainly functions to inhibit the activation and phagocytosis of macrophages through CD47/SIRP $\alpha$ signaling[94]. The remarkable upregulation of CD47 on the SCLC cell surface may be another important immune escape mechanism by inhibiting the activation and phagocytosis of macrophages. Preliminary experiments suggested that blocking CD47 induced macrophage-mediated phagocytosis in SCLC cell lines and mouse xenograft models [95]. Dysregulation of the Fas/FasL signaling pathway in tumors by reducing Fas expression and increasing FasL expression could participate in tumor development and immune escape $[96,97]$. Fas expression is markedly decreased and even completely lost in lung tumors. However, FasL expression is decreased in most NSCLCs but is upregulated in 91\% of SCLCs. FasL overexpression in the context of Fas downregulation in SCLC confers the ability on SCLC cells of inducing paracrine killing of Fas-expressing cytotoxic T cells and inhibiting self-apoptosis. These results indicated that the heavy tumor burden in SCLC makes the immune system less functional than in NSCLC through IL-15 secretion and the CD47/SIRP $\alpha$ and Fas/FasL pathways.

\section{Autocrine and paracrine regulation in SCLC}

A distinguishing feature of SCLC is the substantial autocrine and paracrine stimulation by growth factor receptors and chemokine receptors. Bombesin/gastrin-releasing peptide (BN/GRP) is a relevant growth factor in SCLC, and its receptor in overexpressed in SCLC [98]. Compared with NSCLC, which does not secrete autocrine growth factor granulocyte chemotactic protein (GCP-2), SCLC constantly secretes GCP-2 and expresses its receptors, CXCR1 and CXCR2 [99]. Stem cell factor (SCF) and its receptor c-kit, expressed in 40-70\% SCLC specimens, are important regulators of SCLC viability [100]. In contrast to NSCLC cell lines, SCLC cell lines do not express intercellular adhesion molecule-1 (ICAM-1), which is involved in the interaction with lymphokine-activated killer cells [101]. IL-8 acts as an autocrine and/or paracrine growth factor for lung cancer, mediated by CXCR1 and CXCR2 receptors on the tumor cell surface. However, NSCLC cells produce much higher levels of IL-8 than SCLC cells. As a result, IL-8 may attract neutrophils and induce the immune response in NSCLC but mainly promote autocrine growth in SCLC [102, 103]. Lopez-Gonzalez et al. found that NSCLC and SCLC cell lines had diverse expression levels of TGF- $\beta$ and its receptor [104]. NSCLC synthesizes both TGF- $\beta$ isoforms and TGF- $\beta$ RII. In stark contrast, malignant transformation in SCLC alters the synthesis of TGF- $\beta$ isoforms and TGF- $\beta$ RII, thereby avoiding autocrine and paracrine growth inhibition by TGF- $\beta$ in SCLC cells. Another study found that four to eight SCLC cell lines could constantly secrete biologically active TGF- $\beta 1$ to suppress IL-2-dependent T cell growth, and a specific anti-TGF- $\beta 1$ antibody blocked the immunosuppressive activity induced by the SCLC cell lines [105]. These studies suggested that SCLC cells could markedly promote self-growth via autocrine and paracrine regulation but had less immune stimulatory function in adjacent immune cells compared with NSCLC cells.

These comparative analyses of immune regulation patterns between SCLC and NSCLC partially explain the immunodeficiency observed in patients with SCLC and the worse immune response in SCLC patients. Detailed investigations are essential to determine the exact immune escape mechanisms in SCLC to support the development of immunotherapy for SCLC.

\section{Future of immunotherapy for SCLC}

As demonstrated above, there are many differences underlying immune regulation patterns between NSCLC and SCLC. We note that several potential strategies could possibly improve the clinical outcome of immunotherapy for SCLC.

\section{Chemotherapy and radiotherapy prior to ICBs}

The intrinsic nature of ED-SCLC is so aggressive that a great proportion of patients dropped out or discontinued without completing the whole treatment course [51]. As illustrated in part 3, SCLC cells can induce immunosuppression and promote autocrine and paracrine regulation. Immune suppression seems to be associated with tumor load and could improve upon the reduction of 
the tumor load [93]. Chemotherapy and radiotherapy can not only reduce the heavy tumor burden of SCLC and further reinvigorate immune function but can also elevate PD-L1 expression and tumor antigen presentation by MHC molecules, thus favoring subsequent immunotherapy $[106,107]$. We speculate that chemotherapy and radiotherapy prior to ICBs could be effective in improving the clinical outcome of immunotherapy for SCLC.

\section{Combination of anti-angiogenesis and ICBs}

Angiogenesis is one of the hallmarks of cancer [108]. Anlotinib, an oral anti-angiogenesis tyrosine multikinase inhibitor, is an approved third-line or later therapy for advanced NSCLC according to the Chinese Food and Drug Administration (CFDA), based on the ALTER 0303 study [109]. ALTER 1202, a phase II trial, has also demonstrated that anlotinib significantly improves OS as a third-line or later treatment [110]. Combination therapy with antiangiogenic therapy is a new paradigm in the treatment of advanced-stage malignancies via normalizing tumor vasculature and increasing drug delivery $[111,112]$. Vascular endothelial growth factor (VEGF), the major regulator in tumor angiogenesis, plays an important role in immune modulation by blocking dendritic cell (DC) differentiation, reducing $\mathrm{T}$ cell infiltration, inducing inhibitory immune cells (Tregs and MDSCs) and inhibiting $\mathrm{T}$ cell development $[112,113]$. Theoretically, combining immunotherapy with antiangiogenic treatment may have a synergistic effect, enhance the efficacy of both. Atezolizumab in combination with bevacizumab and chemotherapy significantly improved OS among patients with metastatic nonsquamous NSCLC in IMpower 150 [55]. The combination of ICBs with antiangiogenic therapy could also be promising in SCLC.

\section{Biomarker selection}

Compared with NSCLC, PD-L1 expression is relatively low in SCLC. In NSCLC, multiple clinical trials have defined PD-L1 as a biomarker for pembrolizumab, and the FDA-approved IHC assay utilized cutoffs of a 50\% tumor proportion score for first-line and $1 \%$ for secondline pembrolizumab therapy [114]. Although PD-L1 expression was associated with a survival benefit in SCLC as indicated in early phase studies $[66,71,72]$, it is not a perfect biomarker in SCLC. TMB, another biomarker of immunotherapy, could also predict the clinical outcome of ICBs based on exploratory analyses of CheckMate 026 [39] and CheckMate 227 [76] in NSCLC. In SCLC, Hellmann et al. retrospectively evaluated 211 TMBevaluable patients from the total nonrandomized and randomized cohorts of CheckMate 032 [33]. For patients receiving nivolumab monotherapy and nivolumab plus ipilizumab, the ORR was higher in patients with a high TMB $(21.3 \%$ and $46.2 \%)$ than in patients with a medium
(6.8\% and $16.0 \%)$ or low (4.8\% and $22.2 \%)$ TMB. The OS for SCLC patients with a high TMB treated with nivolumab plus ipilimumab (22 months) was nearly three times that (6-8 months) achieved by the historical topotecan chemotherapy [16, 17]. Additionally, there was no association between PD-L1 expression and TMB in lung cancer $[33,39,115]$. Patients receiving nivolumab with a high level of these two biomarkers had a higher ORR than those with only one of these factors in CheckMate 026 [39]. Up to now, there are no exact biomarkers for immunotherapy in SCLC, TMB seemed to be more important than PD-L1 expression and perhaps the combination of the two biomarkers will be more valuable for selecting patients who will benefit from ICBs.

\section{Conclusion}

SCLC is an aggressive cancer type with poor prognosis. Limitations in the current treatment choices for SCLC give impetus to the search for novel therapeutic approaches including immunotherapy. Despite the similar level and distribution of the TMB between NSCLC and SCLC, the clinical efficacy of ICBs for SCLC is far less pronounced than that for NSCLC. Most studies concerning ICBs in SCLC failed to elicit better clinical outcomes than traditional chemotherapy. The potential mechanisms involved may be (1) low PD-L1 expression in SCLC, (2) downregulation of MHC molecules in SCLC, (3) immunosuppression induced by SCLC cells, and (4) autocrine and paracrine regulation in SCLC. Nevertheless, ICBs also brought about promising clinical benefits in SCLC. Atezolizumab in combination with etoposide and carboplatin prolonged OS by 2 months over chemotherapy alone and has recently received FDA approval as the first-line treatment for SCLC.

How to improve the clinical efficacy of immunotherapy for SCLC is an essential issue. Based on the dysregulation of the immune system in SCLC, we expect that chemotherapy and radiotherapy prior to immunotherapy could be more effective in improving the clinical outcome of ICBs in patients with SCLC. The combination of ICBs with antiangiogenic therapy may be a novel option for SCLC. Last, TMB seemed to be a more valuable biomarker for the efficacy of ICBs in SCLC, and the combination of TMB and PD-L1 may be promising for selecting patients who benefit from immunotherapy in SCLC.

\footnotetext{
Abbreviations

AACR: American Association for Cancer Research; AE: Adverse event; CFDA: Chinese Food and Drug Administration; CTLA4: Cytotoxic Tlymphocyte protein-4; ES-SCLC: Extensive-stage SCLC; FDA: US Food and Drug Administration; G3-4: Grade 3-4; GCP-2: Granulocyte chemotactic protein; ICAM: Intercellular adhesion molecule-1; ICB: Immune checkpoint

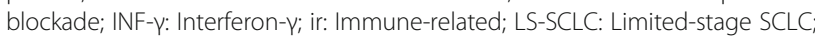
MHC: Major histocompability complex; NSCLC: Non-small cell lung cancer; ORR: Overall response rate; OS: Overall survival; PD-1: Programmed cell death protein-1; PD-L1: Programmed cell death ligand-1; PFS: Progression-free survival; SCF: Stem cell factor; SCLC: Small cell lung cancer; TIL: Tumor
} 
infiltration lymphocyte; TIM: Tumor infiltration macrophage; TMB: Tumor mutation burden; TME: Tumor mutation burden

\section{Acknowledgments}

Not applicable

\section{Authors' contributions}

$\mathrm{HZ}$ and JY designed the study. YT drafted the manuscript. XZ and $\mathrm{AH}$ coordinated the study. All authors read and approved the final manuscript.

\section{Funding}

This work was supported by The Key Research and Development Program of Shandong Province (grant number 2016CYJS01A03).

\section{Availability of data and materials}

The dataset supporting the conclusions of this article is included within the article.

\section{Ethics approval and consent to participate}

Not applicable

\section{Consent for publication}

Not applicable

\section{Competing interests}

The authors declare that they have no competing interests.

\section{Author details}

${ }^{1}$ Department of Radiation Oncology, Shandong Cancer Hospital and Institute, Shandong University, Jinan, China. 'Department of Radiation Oncology, Shandong First Medical University and Shandong Academy of Medical Sciences, Jinan, China. ${ }^{3}$ Department of Radiation Oncology, Shandong Cancer Hospital and Institute, Shandong First Medical University and Shandong Academy of Medical Sciences, 440 Jiyan Road, Jinan 250117 China.

Received: 5 April 2019 Accepted: 14 June 2019

\section{Published online: 28 June 2019}

\section{References}

1. Bray F, Ferlay J, Soerjomataram I, et al. Global cancer statistics 2018 GLOBOCAN estimates of incidence and mortality worldwide for 36 cancers in 185 countries. CA Cancer J Clin. 2018;68:394-424.

2. National Cancer Institute. SEER cancer statistics review,1975-2014,https:// seer.cancer.gov/archive/csr/1975_2014/.

3. American Cancer Society. Cancer facts \& figures 2014. Atlanta, GA: American Cancer Society; 2014

4. Pesch B, Kendzia B, Gustavsson P, et al. Cigarette smoking and lung cancerrelative risk estimates for the major histological types from a pooled analysis of case-control studies. Int J Cancer. 2012;131:1210-9.

5. Varghese AM, Zakowski MF, Yu HA, et al. Small-cell lung cancers in patients who never smoked cigarettes. J Thorac Oncol. 2014;9:892-6.

6. Khuder SA. Effect of cigarette smoking on major histological types of lung cancer: a meta-analysis. Lung Cancer (Amsterdam, Netherlands). 2001:31:139-48.

7. Alexandrov LB, Nik-Zainal S, Wedge DC, et al. Signatures of mutational processes in human cancer. Nature. 2013;500:415-21.

8. Peifer M, Fernandez-Cuesta L, Sos ML, et al. Integrative genome analyses identify key somatic driver mutations of small-cell lung cancer. Nat Genet. 2012;44:1104-10.

9. George J, Lim JS, Jang SJ, et al. Comprehensive genomic profiles of small cell lung cancer. Nature. 2015;524:47-53.

10. Pleasance ED, Stephens PJ, O'Meara S, et al. A small-cell lung cancer genome with complex signatures of tobacco exposure. Nature. 2010; 463:184-90

11. National Comprehensive Cancer Network. Clinical practice guidelines in oncology. Small cell lung cancer. 2019; Version 1

12. Zatloukal P, Cardenal F, Szczesna A, et al. A multicenter internationa randomized phase III study comparing cisplatin in combination with irinotecan or etoposide in previously untreated small-cell lung cancer patients with extensive disease. Ann Oncol. 2010;21:1810-6.
13. Hanna N, Bunn PA Jr, Langer C, et al. Randomized phase III trial comparing irinotecan/cisplatin with etoposide/cisplatin in patients with previously untreated extensive-stage disease small-cell lung cancer. J Clin Oncol. 2006;24:2038-43.

14. Schmittel A, Sebastian M, Fischer von Weikersthal L, et al. A German multicenter, randomized phase III trial comparing irinotecan-carboplatin with etoposide-carboplatin as first-line therapy for extensive-disease smallcell lung cancer. Ann Oncol. 2011;22:1798-804.

15. Byers LA, Rudin CM. Small cell lung cancer: where do we go from here? Cancer. 2015;121:664-72.

16. von Pawel J, Jotte R, Spigel DR, et al. Randomized phase III trial of amrubicin versus topotecan as second-line treatment for patients with small-cell lung cancer. J Clin Oncol. 2014;32:4012-9.

17. O'Brien ME, Ciuleanu TE, Tsekov $H$, et al. Phase III trial comparing supportive care alone with supportive care with oral topotecan in patients with relapsed small-cell lung cancer. J Clin Oncol. 2006;24:5441-7.

18. Cheng S, Evans WK, Stys-Norman D, et al. Chemotherapy for relapsed small cell lung cancer: a systematic review and practice guideline. J Thorac Oncol. 2007;2:348-54

19. Vesely MD, Kershaw MH, Schreiber RD, et al. Natural innate and adaptive immunity to cancer. Annu Rev Immunol. 2011;29:235-71.

20. Chen DS, Mellman I. Elements of cancer immunity and the cancer-immune set point. Nature. 2017;541:321-30.

21. Ribas A, Wolchok JD. Cancer immunotherapy using checkpoint blockade. Science. 2018;359:1350-5.

22. Chen DS, Mellman I. Oncology meets immunology: the cancer-immunity cycle. Immunity. 2013;39:1-10.

23. Rowshanravan B, Halliday N, Sansom DM. CTLA-4: a moving target in immunotherapy. Blood. 2018;131:58-67.

24. Hodi FS, O'Day SJ, McDermott DF, et al. Improved survival with ipilimumab in patients with metastatic melanoma. N Engl J Med. 2010;363:711-23.

25. Robert C, Thomas L, Bondarenko I, et al. Ipilimumab plus dacarbazine for previously untreated metastatic melanoma. N Engl J Med. 2011;364:2517-26.

26. Sun C, Mezzadra R, Schumacher TN. Regulation and function of the PD-L1 Checkpoint. Immunity. 2018;48:434-52.

27. Topalian SL, Drake CG, Pardoll DM. Immune checkpoint blockade: a common denominator approach to cancer therapy. Cancer Cell. 2015;27:450-61.

28. Boussiotis VA. Molecular and biochemical aspects of the PD-1 checkpoint pathway. N Engl J Med. 2016;375:1767-78.

29. Topalian SL, Taube JM, Anders RA, et al. Mechanism-driven biomarkers to guide immune checkpoint blockade in cancer therapy. Nat Rev Cancer. 2016;16:275-87.

30. Sanmamed MF, Chen L. A paradigm shift in cancer immunotherapy: from enhancement to normalization. Cell. 2018;175:313-26.

31. Hargadon KM, Johnson CE, Williams CJ. Immune checkpoint blockade therapy for cancer: an overview of FDA-approved immune checkpoint inhibitors. Int Immunopharmacol. 2018:62:29-39.

32. Sabari JK, Lok BH, Laird JH, et al. Unravelling the biology of SCLC: implications for therapy. Nat Rev Clin Oncol. 2017:14:549-61.

33. Hellmann MD, Callahan MK, Awad MM, et al. Tumor mutational burden and efficacy of nivolumab monotherapy and in combination with ipilimumab in small-cell lung cancer. Cancer Cell. 2018;33:853-61.

34. Coulie PG, Van den Eynde BJ, van der Bruggen $P$, et al. Tumour antigens recognized by $T$ lymphocytes: at the core of cancer immunotherapy. Nat Rev Cancer. 2014:14:135-46.

35. Chan TA, Yarchoan M, Jaffee $E$, et al. Development of tumor mutation burden as an immunotherapy biomarker: utility for the oncology clinic. Ann Oncol. 2019;30:44-56.

36. McGranahan N, Furness AJ, Rosenthal R, et al. Clonal neoantigens elicit $T$ cell immunoreactivity and sensitivity to immune checkpoint blockade. Science. 2016;351:1463-9.

37. Rizvi NA, Hellmann MD, Snyder A, et al. Cancer immunology. Mutational landscape determines sensitivity to PD-1 blockade in non-small cell lung cancer. Science. 2015:348:124-8.

38. Cristescu R, Mogg R, Ayers M, et al. Pan-tumor genomic biomarkers for PD-1 checkpoint blockade-based immunotherapy. Science. 2018;362.

39. Carbone DP, Reck M, Paz-Ares L, et al. First-line nivolumab in stage IV or recurrent non-small-cell lung cancer. N Engl J Med. 2017;376:2415-26.

40. Wang W, Hodkinson P, McLaren F, et al. Histologic assessment of tumorassociated CD45(+) cell numbers is an independent predictor of prognosis in small cell lung cancer. Chest. 2013;143:146-51. 
41. Berghoff AS, Ricken G, Wilhelm D, et al. Tumor infiltrating lymphocytes and PD-L1 expression in brain metastases of small cell lung cancer (SCLC). J Neurooncol. 2016;130:19-29.

42. Eerola AK, Soini Y, Paakko P. A high number of tumor-infiltrating lymphocytes are associated with a small tumor size, low tumor stage, and a favorable prognosis in operated small cell lung carcinoma. Clin Cancer Res. 2000;6:1875-81

43. Studnicka M, Wirnsberger R, Neumann M, et al. Peripheral blood lymphocyte subsets and survival in small-cell lung cancer. Chest. 1994;105:1673-8.

44. Xie D, Marks R, Zhang M, et al. Nomograms predict overall survival for patients with small-cell lung cancer incorporating pretreatment peripheral blood markers. J Thorac Oncol. 2015;10:1213-20.

45. Tian T, Gu X, Zhang B, et al. Increased circulating CD14(+)HLA-DR-/low myeloid-derived suppressor cells are associated with poor prognosis in patients with small-cell lung cancer. Cancer Biomarkers. 2015;15:425-32.

46. Zhou T, Zhan J, Hong S, et al. Ratio of C-reactive protein/albumin is an inflammatory prognostic score for predicting overall survival of patients with small-cell lung cancer. Scientific reports. 2015;5:10481.

47. Wang W, Hodkinson P, McLaren F, et al. Small cell lung cancer tumour cells induce regulatory $T$ lymphocytes, and patient survival correlates negatively with FOXP3+ cells in tumour infiltrate. Int J Cancer. 2012;131:E928-37.

48. Lynch TJ, Bondarenko I, Luft A, et al. Ipilimumab in combination with paclitaxel and carboplatin as first-line treatment in stage IIIB/IV non-smallcell lung cancer: results from a randomized, double-blind, multicenter phase II study. J Clin Oncol. 2012;30:2046-54.

49. Reck M, Bondarenko I, Luft A, et al. Ipilimumab in combination with paclitaxel and carboplatin as first-line therapy in extensive-disease-small-cell lung cancer: results from a randomized, double-blind, multicenter phase 2 trial. Ann Oncol. 2013;24:75-83.

50. Govindan R, Szczesna A, Ahn MJ, et al. Phase III trial of ipilimumab combined with paclitaxel and carboplatin in advanced squamous nonsmall-cell lung cancer. J Clin Oncol. 2017;35:3449-57.

51. Reck M, Luft A, Szczesna A, et al. Phase III randomized trial of ipilimumab plus etoposide and platinum versus placebo plus etoposide and platinum in extensive-stage small-cell lung cancer. J Clin Oncol. 2016;34:3740-8.

52. Jotte RMCF, Vynnychenko I, et al. IMpower131: Primary PFS and safety analysis of a randomized phase III study of atezolizumab + carboplatin +paclitaxel or nab-paclitaxel vs carboplatin + nab-paclitaxel as $1 \mathrm{~L}$ therapy in advanced squamous NSCLC. J Clin Oncol. 2018;36(18_suppl):LBA9000.

53. Horn L, Mansfield AS, Szczesna A, et al. First-line atezolizumab plus chemotherapy in extensive-stage small-cell lung cancer. N Engl J Med. 2018;379:2220-9

54. Barlesi F, MN MC, et al. IMpower 132: Atezolizumab in combination with pemetrexed and platinum-based chemotherapy in advanced nonsquamous NSCLC: WCLC; 2018.

55. Socinski MA, Jotte RM, Cappuzzo F, et al. Atezolizumab for first-line treatment of metastatic nonsquamous NSCLC. N Engl J Med. 2018;378:2288-301.

56. Brahmer J, Reckamp KL, Baas $\mathrm{P}$, et al. Nivolumab versus docetaxel in advanced squamous-cell non-small-cell lung cancer. N Engl J Med. 2015;373:123-35.

57. Antonia SJ, Lopez-Martin JA, Bendell J, et al. Nivolumab alone and nivolumab plus ipilimumab in recurrent small-cell lung cancer (CheckMate 032): a multicentre, open-label, phase 1/2 trial. Lancet Oncol. 2016;17:883-95.

58. Borghaei $H$, Paz-Ares L, Horn L, et al. Nivolumab versus docetaxel in advanced nonsquamous non-small-cell lung cancer. $\mathrm{N}$ Engl J Med. 2015;373:1627-39.

59. Reck MVD, Ciuleanu T, et al. Efficacy and safety of nivolumab (nivo) monotherapy versus chemotherapy (chemo) in recurrent small cell lung cancer (SCLC): Results from CheckMate 331: ESMO; 2018.

60. Herbst RS, Baas P, Kim DW, et al. Pembrolizumab versus docetaxel for previously treated, PD-L1-positive, advanced non-small-cell lung cancer (KEYNOTE-010): a randomised controlled trial. Lancet (London, England). 2016:387:1540-50

61. Chung HC P-PS, Lopez-Martin J,et al. Pembrolizumab after two or more lines of prior therapy in patients with advanced small-cell lung cancer (SCLC):results from the KEYNOTE-028 and KEYNOTE-158 studies. AACR 2019; abstract CT073.

62. Fehrenbacher $L$, Spira A, Ballinger $M$, et al. Atezolizumab versus docetaxel for patients with previously treated non-small-cell lung cancer (POPLAR): a multicentre, open-label, phase 2 randomised controlled trial. Lancet (London, England). 2016;387:1837-46.
63. Pujol JL, Greillier L, Audigier-Valette C, et al. A randomized non-comparative phase 2 study of anti-programmed cell death-ligand 1 atezolizumab or chemotherapy as second-line therapy in patients with small cell lung cancer: results from the IFCT-1603 trial. J Thorac Oncol. 2019.

64. Rittmeyer A, Barlesi F, Waterkamp D, et al. Atezolizumab versus docetaxel in patients with previously treated non-small-cell lung cancer (OAK): a phase 3, open-label, multicentre randomised controlled trial. Lancet (London, England). 2017;389:255-65.

65. Antonia SJ, Villegas A, Daniel D, et al. Overall survival with durvalumab after chemoradiotherapy in stage III NSCLC. N Engl J Med. 2018;379:2342-50.

66. Gadgeel SM, Pennell NA, Fidler MJ, et al. Phase II study of maintenance pembrolizumab in patients with extensive-stage small cell lung cancer (SCLC). J Thorac Oncol. 2018;13:1393-9.

67. Arriola E, Wheater M, Galea I, et al. Outcome and biomarker analysis from a multicenter phase 2 study of ipilimumab in combination with carboplatin and etoposide as first-line therapy for extensive-stage SCLC. J Thorac Oncol. 2016;11:1511-21.

68. Paz-Ares L, Luft A, Vicente D, et al. Pembrolizumab plus chemotherapy for squamous non-small-cell lung cancer. N Engl J Med. 2018;379:2040-51.

69. Gandhi L, Rodriguez-Abreu D, Gadgeel S, et al. Pembrolizumab plus chemotherapy in metastatic non-small-cell lung cancer. N Engl J Med. 2018;378:2078-92

70. Weiss GJ, Waypa J, Blaydorn L, et al. A phase lb study of pembrolizumab plus chemotherapy in patients with advanced cancer (PembroPlus). Br J Cancer. 2017;117:33-40.

71. Ott PA, Elez E, Hiret $\mathrm{S}$, et al. Pembrolizumab in patients with extensive-stage small-cell lung cancer: results from the phase Ib KEYNOTE-028 Study. J Clin Oncol. 2017:35:3823-9.

72. Chung HCL-MJ, Kao SCH, Miller WH, Ros W, Gao B. Phase 2 study of pembrolizumab in advanced small-cell lung cancer (SCLC): KEYNOTE-158. J Clin Oncol. 2018;15(36):8506

73. Patel SP, Kurzrock R. PD-L1 expression as a predictive biomarker in cancer immunotherapy. Mol Cancer Ther. 2015;14:847-56.

74. Sacher AG, Gandhi L. biomarkers for the clinical use of PD-1/PD-L1 inhibitors in non-small-cell lung cancer: a review. JAMA Oncol. 2016;2:1217-22.

75. Kerr KM, Tsao MS, Nicholson AG, et al. Programmed death-ligand 1 immunohistochemistry in lung cancer: in what state is this art? J Thorac Oncol. 2015;10:985-9.

76. Hellmann MD, Ciuleanu TE, Pluzanski A, et al. Nivolumab plus ipilimumab in lung cancer with a high tumor mutational burden. N Engl J Med. 2018;378:2093-104.

77. Inamura K, Yokouchi Y, Kobayashi M, et al. Relationship of tumor PD-L1 (CD274) expression with lower mortality in lung high-grade neuroendocrine tumor. Cancer Med. 2017;6:2347-56.

78. Yasuda Y, Ozasa H, Kim YH. PD-L1 expression in small cell lung cancer. J Thorac Oncol. 2018;13:e40-1.

79. Schultheis AM, Scheel AH, Ozretic L, et al. PD-L1 expression in small cell neuroendocrine carcinomas. Eur J Cancer. 2015;51:421-6.

80. Yu H, Batenchuk C, Badzio A, et al. PD-L1 Expression by two complementary diagnostic assays and mRNA in situ hybridization in small cell lung cancer. J Thorac Oncol. 2017;12:110-20.

81. Kim HS, Lee JH, Nam SJ, et al. Association of PD-L1 expression with tumorinfiltrating immune cells and mutation burden in high-grade neuroendocrine carcinoma of the lung. J Thorac Oncol. 2018;13:636-48.

82. Ishii H, Azuma K, Kawahara A, et al. Significance of programmed cell deathligand 1 expression and its association with survival in patients with small cell lung cancer. J Thorac Oncol. 2015;10:426-30.

83. Komiya T, Madan R. PD-L1 expression in small cell lung cancer. Eur J Cancer. 2015:51:1853-5.

84. Turley SJ, Cremasco V, Astarita JL. Immunological hallmarks of stromal cells in the tumour microenvironment. Nat Rev Immunol. 2015;15:669-82.

85. Tang H, Liang Y, Anders RA, et al. PD-L1 on host cells is essential for PD-L1 blockade-mediated tumor regression. J Clin Invest. 2018;128:580-8.

86. Lin H, Wei S, Hurt EM, et al. Host expression of PD-L1 determines efficacy of PDL1 pathway blockade-mediated tumor regression. J Clin Invest. 2018;128:805-15.

87. Doyle A. Markedly decreased expression of class I histocompatibility antigens, protein, and mRNA in human small-cell lung cancer. J Exp Med. 1985;161:1135-51.

88. Traversari C, Meazza R, Coppolecchia M, et al. IFN-gamma gene transfer restores HLA-class I expression and MAGE-3 antigen presentation to CTL in HLA-deficient small cell lung cancer. Gene Therapy. 1997:4:1029-35. 
89. He Y, Rozeboom L, Rivard CJ, et al. MHC class II expression in lung cancer. Lung Cancer (Amsterdam, Netherlands). 2017;112:75-80.

90. Yazawa T, Ito T, Kamma H, et al. Complicated mechanisms of class II transactivator transcription deficiency in small cell lung cancer and neuroblastoma. Am J Pathol. 2002;161:291-300.

91. Yazawa T, Kamma H, Fujiwara M, et al. Lack of class II transactivator causes severe deficiency of HLA-DR expression in small cell lung cancer. J Pathol. 1999;187:191-9.

92. Masuno T, Ikeda T, Yokota S, et al. Immunoregulatory T-lymphocyte functions in patients with small cell lung cancer. Cancer Res. 1986; 46:4195-9.

93. Fischer JR, Schindel M, Stein N, et al. Selective suppression of cytokine secretion in patients with small-cell lung cancer. Ann Oncol. 1995;6:921-6.

94. Matozaki T, Murata Y, Okazawa H, et al. Functions and molecular mechanisms of the CD47-SIRPalpha signalling pathway. Trends Cell Biol. 2009;19:72-80

95. Weiskopf K, Jahchan NS, Schnorr PJ, et al. CD47-blocking immunotherapies stimulate macrophage-mediated destruction of small-cell lung cancer. J Clin Invest. 2016;126:2610-20

96. Viard-Leveugle I, Veyrenc $S$, French LE, et al. Frequent loss of Fas expression and function in human lung tumours with overexpression of FasL in small cell lung carcinoma. J Pathol. 2003;201:268-77.

97. Hopkins-Donaldson S, Ziegler A, Kurtz S, et al. Silencing of death receptor and caspase-8 expression in small cell lung carcinoma cell lines and tumors by DNA methylation. Cell Death Differ. 2003;10:356-64.

98. Zhou J, Chen J, Mokotoff M, et al. Bombesin/gastrin-releasing peptide receptor: a potential target for antibody-mediated therapy of small cell lung cancer. Clin Cancer Res. 2003;9:4953-60.

99. Zhu YM, Bagstaff SM, Woll PJ. Production and upregulation of granulocyte chemotactic protein-2/CXCL6 by IL-1beta and hypoxia in small cell lung cancer. Br J Cancer. 2006;94:1936-41.

100. Kijima T, Maulik G, Ma PC, et al. Regulation of cellular proliferation, cytoskeletal function, and signal transduction through CXCR4 and c-Kit in small cell lung cancer cells. Cancer Res. 2002;62:6304-11.

101. Melis M, Spatafora M, Melodia A, et al. ICAM-1 expression by lung cancer cell lines: effects of upregulation by cytokines on the interaction with LAK cells. Eur Respir J. 1996;9:1831-8.

102. Zhu YM, Webster SJ, Flower D, et al. Interleukin-8/CXCL8 is a growth factor for human lung cancer cells. Br J Cancer. 2004;91:1970-6.

103. Shibakura M, Niiya K, Kiguchi T, et al. Induction of IL-8 and monoclyte chemoattractant protein-1 by doxorubicin in human small cell lung carcinoma cells. Int J Cancer. 2003;103:380-6.

104. Lopez-Gonzalez JS, Aguilar-Cazares D, Prado-Garcia H, et al. Lack of correlation between growth inhibition by TGF-beta and the percentage of cells expressing type II TGF-beta receptor in human non-small cell lung carcinoma cell lines. Lung Cancer (Amsterdam, Netherlands). 2002;38:149-58

105. Fischer JR, Darjes $H$, Lahm H, et al. Constitutive secretion of bioactive transforming growth factor beta 1 by small cell lung cancer cell lines. Eur J Cancer. 1994;30a:2125-9.

106. Ko EC, Raben D, Formenti SC. The integration of radiotherapy with immunotherapy for the treatment of non-small cell lung cancer. Clin Cancer Res. 2018;24:5792-806.

107. Zitvogel L, Apetoh L, Ghiringhelli F, et al. Immunological aspects of cancer chemotherapy. Nat Rev Immunol. 2008;8:59-73.

108. Hanahan D, Weinberg RA. The hallmarks of cancer. Cell. 2000;100:57-70.

109. Han B, Li K, Wang Q, et al. Effect of anlotinib as a third-line or further treatment on overall survival of patients with advanced non-small cell lung cancer: the ALTER 0303 phase 3 randomized clinical trial. JAMA Oncol. 2018;4:1569-75.

110. Cheng Y WQ, Li K, Shi J, Wu L, Han B, et al. Anlotinib as third-line or furtherline treatment in relapsed SCLC: a multicentre, randomized, double-blind phase 2 trial. . WCLC 2018; OA 13.03.

111. Jain RK. Normalizing tumor vasculature with anti-angiogenic therapy: a new paradigm for combination therapy. Nature Med. 2001;7:987-9.

112. Ellis LM, Hicklin DJ. VEGF-targeted therapy: mechanisms of anti-tumour activity. Nat Rev Cancer. 2008:8:579.

113. Manegold C, Dingemans AC, Gray JE, et al. The potential of combined immunotherapy and antiangiogenesis for the synergistic treatment of advanced NSCLC. J Thorac Oncol. 2017;12:194-207.
114. National Comprehensive Cancer Network. Clinical practice guidelines in oncology. Non-small cell lung cancer. 2019; Version 3.

115. Hellmann MD, Nathanson T, Rizvi H, et al. Genomic features of response to combination immunotherapy in patients with advanced non-small-cell lung cancer. Cancer Cell. 2018;33:843-852 e844.

\section{Publisher's Note}

Springer Nature remains neutral with regard to jurisdictional claims in published maps and institutional affiliations.
Ready to submit your research? Choose BMC and benefit from:

- fast, convenient online submission

- thorough peer review by experienced researchers in your field

- rapid publication on acceptance

- support for research data, including large and complex data types

- gold Open Access which fosters wider collaboration and increased citations

- maximum visibility for your research: over $100 \mathrm{M}$ website views per year

At BMC, research is always in progress.

Learn more biomedcentral.com/submissions 